\title{
STMN-1 is a potential marker of lymph node metastasis in distal esophageal adenocarcinomas and silencing its expression can reverse malignant phenotype of tumor cells
}

\author{
Javed Akhtar ${ }^{1,2}$, Zhou Wang ${ }^{1}$, Che Yu ${ }^{3}$, Chen-Sheng Li ${ }^{2}$ Yu-Long Shi ${ }^{*}$ and Hong-Jun Liu ${ }^{2^{*}}$
}

\begin{abstract}
Background: Distal esophageal adenocarcinoma is a highly aggressive neoplasm. Despite advances in diagnosis and therapy, the prognosis is still poor. Stathmin (STMN-1) is a ubiquitously expressed microtubule destabilizing phosphoprotein. It promotes the disassembly of microtubules and prevents assembly. STMN-1 can cause uncontrolled cell proliferation when mutated and not functioning properly. Recently, found to be overexpressed in many types of human cancers. However, its clinical significance remains elusive in distal esophageal adenocarcinoma. Here, we reported for the first time that STMN-1 is highly overexpressed in adenocarcinomas of the distal esophagus and strongly associated with lymph node metastasis.
\end{abstract}

Methods: STMN-1 expression in 63 cases of distal esophageal adenocarcinoma was analyzed by immunoblotting, while expression in esophageal adenocarcinoma cells was determined by immunocytochemistry, immunofluorescence, qRT-PCR and western blotting. Lentivirus-mediated RNAi was employed to knock-down STMN-1 expression in Human esophageal adenocarcinoma cells. The relationship between STMN-1 expression and lymph node metastasis in distal esophageal adenocarcinoma was determined by univariate and multivariate analyses.

Results: STMN-1 was detected in 31 (49.21\%) of the 63 cases. STMN-1 was highly overexpressed in specimens with lymph node metastasis pN (+), but its expression was almost undetected in $\mathrm{pN}(-)$ status. Multivarian regression analysis demonstrated that STMN-1 overexpression is an independent factor for lymph node metastasis in distal esophageal adenocarcinoma. STMN-1 shRNA effectively reduced STMN-1 expression in esophageal adenocarcinoma cells $(P<0.05)$, which significantly suppressed proliferation $(P<0.05)$, increased migration $(P<0.05)$ and invasion ability $(P<0.05)$ and $G 1$ phase arrest $(P<0.05)$ which lead to induction of apoptosis in esophageal adenocarcinoma cells in vitro. To verify the in vitro data, we conducted in vivo tumor xenograft studies. Esophageal adenocarcinoma cells stably transfected with STMN-1 shRNA significantly reduced tumor xenografts volume in vivo.

Conclusions: STMN-1 overexpression is associated with lymph node metastasis and increase malignancy in distal esophageal adenocarcinoma. In vivo and in vitro laboratory findings, suggests that STMN-1 may be a suitable target for future therapeutic strategies in distal esophageal adenocarcinoma.

Keywords: Stathmin1, Distal esophageal adenocarcinoma, Short hairpin RNA, Multivariate logistic regression

\footnotetext{
*Correspondence: shiyl9889@126.com; liuhongjun502@hotmail.com

${ }^{2}$ Division of Gastrointestinal Surgery, Provincial Hospital Affiliated to

Shandong University, 250021 Shandong, China

Full list of author information is available at the end of the article
} 


\section{Background}

Distal esophageal adenocarcinoma is a highly aggressive neoplasm. Despite advances in diagnosis and therapy, the prognosis of the patients is still poor. An estimated 16,640 new cases of esophageal cancer were diagnosed in 2010 in the United States with an estimated 14,500 deaths [1] and an estimated 482,300 new cases and 406,800 deaths occurred in 2008 worldwide [2]. The World Health Organization (WHO) predicts that by 2020, approximately $60 \%$ of all new cancer cases will occur in the least developed nations [3]. Most of the distal esophageal adenocarcinomas are of gastro-genic origin, which superiorly invades the lower part of the esophagus or derived from the malignant degeneration of Barrett's esophagus. High prevalence of esophageal squamous cell carcinoma with poor prognosis has been well documented in Chinese population. On clinical basis, distal esophageal adenocarcinoma is also a disease very commonly seen by the thoracic surgeon. Perhaps due to obscure definition of Gastric cardiac cancer and the lack of clear definition of distal esophageal adenocarcinomas; the reports are not commonly seen. In the 2009 UICC/AJCC TNM Classification of Malignant Tumors, Esophageal cancer is redefined as "Any tumor whose epicenter is in the lower esophagus, gastroesophageal junction or proximal $5 \mathrm{~cm}$ of the stomach that extends into the gastroesophageal junction (GEJ) or esophagus" [4,5]. In the past, there were no standard guidelines for distal esophageal adenocarcinomas lymph node clearance.

Adenocarcinomas of the distal esophagus have a high propensity of lymph node metastasis and transcending mucosal spread of disease. These are the main factors limiting the curative potential of surgical treatment. Therefore, investigating molecular biomarker to predict locally advanced tumor with lymph node metastasis is significant in the clinical practice. The current data showed us there are no known identifiable molecular biological markers in the early detection of metastasis in adenocarcinoma of the distal esophagus. An understanding of the molecular basis for the development of the distal esophageal adenocarcinoma is required to develop effective clinical diagnostic and management strategies.

Surgical resection is the mainstay of therapy for esophageal carcinoma; most patients are diagnosed at an unresectable stage, particularly those with adenocarcinoma of the distal esophagus. Given the poor prognosis and the fact that most are diagnosed at a more advanced or unresectable stage, new therapeutic strategies, treatment options, and novel therapeutic targets are desperately needed. Drug development has been transformed with the identification of and ability to direct treatment at specific molecular targets. In distal esophageal adenocarcinoma, development of novel targeted treatments is lacking.
Stathmin 1 (STMN-1), also known as p17, p18, p19, $19 \mathrm{~K}$, metablastin, oncoprotein 18, LAP 18 and Op18, is a $19 \mathrm{kDa}$ cytosolic protein. Its function as an important regulatory protein of microtubule dynamics has been well characterized [6]. STMN-1 likely plays an important role in cell cycle progression and cell migration. It has been reported that STMN-1 is overexpressed in many human malignancies, such as leukemia, lymphoma, neuroblastoma, ovarian, prostatic, breast and lung cancers [7] and the modulation of its expression correlates with Invasion and metastasis. STMN-1 gene expression in cancer cells has been associated with their proliferation and metastasis $[8,9]$. However, to our knowledge, no study has examined the clinical significance of STMN-1 expression in adenocarcinoma of the distal esophagus.

The present study was initiated to evaluate STMN-1 expression in relation to lymph node metastasis in the adenocarcinoma of the distal esophagus. The result showed that STMN-1 expression correlates with lymph node metastasis.

Multivariate logistic regression analysis demonstrated that STMN-1 overexpression was an independent factor of lymph node metastasis in distal esophageal adenocarcinoma. While we were confirming our clinical findings through laboratory experiments, we also investigated the function of STMN-1 gene in tumorgenesis and evaluated the potential of STMN-1 as a therapeutic target for distal esophageal adenocarcinoma treatment using RNA interference strategy.

\section{Results}

\section{Patient characteristics}

For this study, specimens were examined from a total of 63 patients with resected distal esophageal adenocarcinoma (Table 1). Patients ranged in age from $<40$ years to $\geq 40$ years and the median age was 57 years. Forty five were male (71.43\%) and 18 were female (28.57\%). STMN-1 expression was detected in 31 (49.21\%) of the 63 distal esophageal adenocarcinoma cases. Twenty nine patients (46\%) were diagnosed with $\mathrm{pN} 1, \mathrm{~N} 2$ stage and 34 patients (54\%) were diagnosed with pN0 stage. Twenty one patients (33.33\%) were diagnosed with $\mathrm{T} 1$, T2 stage and forty two patients (66.66\%) with T3, T4a stage (Table 1$)$.

\section{STMN-1 expression in tumor samples}

STMN-1 protein expression was analyzed in the distal esophageal adenocarcinomas tissue in 63 patients. Western blot analysis of samples showed that STMN-1 was up regulated in 31 (49.21\%) of the 63 cases. Twenty one patients $(33.33 \%)$ with $\mathrm{pN} 1$ and $\mathrm{pN} 2$ were found positive for STMN-1 and ten patients (15.87\%) with pN0 also showed overexpression of STMN-1 (Table 1). 
Table 1 Clinicopathological characteristics of 63 distal esophageal adenocarcinoma patients with STMN1 overexpression

\begin{tabular}{|c|c|c|c|c|}
\hline Characteristic & $n=63$ & $\begin{array}{c}\text { STMN1 } \\
\text { overexpression }\end{array}$ & $x^{2}$ & p-Value \\
\hline \multicolumn{5}{|l|}{ Gender } \\
\hline Male & 45 & 23 & 0.229 & 0.633 \\
\hline Female & 18 & 8 & & \\
\hline \multicolumn{5}{|l|}{ Age at surgery } \\
\hline$<40$ years & 8 & 5 & 0.648 & 0.421 \\
\hline$\geq 40$ years & 55 & 26 & & \\
\hline \multicolumn{5}{|l|}{ Weight loss } \\
\hline$\geq 5 \mathrm{~kg}$ & 14 & 8 & 0.454 & 0.501 \\
\hline$<5 \mathrm{~kg}$ & 49 & 23 & & \\
\hline \multicolumn{5}{|l|}{ Grade } \\
\hline Mild, High & 48 & 22 & 0.918 & 0.338 \\
\hline Low & 15 & 9 & & \\
\hline \multicolumn{5}{|l|}{ Stage $(T)$} \\
\hline $\mathrm{T} 1$ & 21 & 5 & 8.129 & $0.004^{a}$ \\
\hline T3, T4a & 42 & 26 & & \\
\hline \multicolumn{5}{|c|}{ Tumor greatest dimension } \\
\hline$<3 \mathrm{~cm}$ & 13 & 4 & 2.228 & 0.136 \\
\hline$\geq 3 \mathrm{~cm}$ & 50 & 27 & & \\
\hline \multicolumn{5}{|l|}{ Stage $(N)$} \\
\hline NO & 34 & 10 & 11.579 & $0.001^{\mathrm{a}}$ \\
\hline $\mathrm{N} 1,2$ & 29 & 21 & & \\
\hline
\end{tabular}

${ }^{\mathrm{a}}$ Statistically significant.

STMN-1 status and its correlation with patient clinicopathological characteristics

Next, we investigated the association between STMN-1 expression and the clinicopathological characteristics of distal esophageal adenocarcinoma cases and found that STMN-1 overexpression was significantly associated with $\mathrm{pN}$ classification $(P=0.001)$. The expression of the STMN-1 protein in distal esophageal adenocarcinoma in the pN1-pN2 stage was significantly higher than in the
pN0 stage and in the T3-T4a stage, it was significantly higher than in the T1-T2 stage. However, no significant correlation was found between STMN-1 expression and gender, age, or degree of differentiation (Table 1).

\section{STMN-1 expression positively correlates with $\mathrm{pN}$ stage in} adenocarcinoma of the distal esophagus

The relationship between STMN-1 status and clinicopathological features was investigated by univariate analysis. STMN-1 protein expression was higher in node positive vs. node negative specimen $(\mathrm{P}=0.001)$ and locally advanced $(\mathrm{T} 3+\mathrm{T} 4 \mathrm{a})$ versus minimally advanced $(\mathrm{T} 1+\mathrm{T} 2)$ distal esophageal adenocarcinoma $(P=0.004)$ but there was no positive correlation with other clinicopathological characteristics. Univariate analysis showed that STMN-1 gene expression is related to $\mathrm{pN}$ stage, and $\mathrm{T}$ classification in distal esophageal adenocarcinoma. Multivariate analysis revealed that only the $\mathrm{pN}$ stage is a significant factor, $(P=0.013)$ as shown in Table 2.

\section{STMN-1 expression in esophageal adenocarcinoma cell lines}

In the current study, we evaluated the state of STMN-1 protein expression in tissue samples by Western blot analysis, (Figure 1A).

We further assessed the level of STMN-1 protein expression in esophageal adenocarcinoma derived cell lines by immunocytochemistry (Figure 1B, C) and immunofluorescence analysis (Figure 1D, E). Strong immunoreactivity of STMN-1 protein was detected in the cytoplasm of esophageal adenocarcinoma cells.

\section{Transfection \& transduction efficiency}

Successful packing of lentivirus was done in HEK 293 T. STMN-1 shRNA transfected cells and control shown in (Figure 2A, B). To determines the efficacies of viral vectors, viral supernatants prepared from either nonsilencing-shRNA (control) or STMN-1 shRNA were added to esophageal adenocarcinoma cells. GFP expression in photomicrographs of STMN-1-shRNA cells and control

Table 2 Multivariate logistic regression analysis

\begin{tabular}{|c|c|c|c|c|c|c|c|c|}
\hline \multirow[b]{2}{*}{ Factors } & \multirow[t]{2}{*}{ B } & \multirow[t]{2}{*}{ S.E. } & \multirow[t]{2}{*}{ Wald } & \multirow[t]{2}{*}{ df } & \multirow[t]{2}{*}{ Sig. } & \multirow[t]{2}{*}{$\operatorname{Exp}(B)$} & \multicolumn{2}{|c|}{ 95.0\% C.I. for EXP(B) } \\
\hline & & & & & & & Lower & Upper \\
\hline Gender & 0.000 & 0.674 & 0.000 & 1 & 1.000 & 1.000 & 0.267 & 3.743 \\
\hline Age & 1.294 & 0.952 & 1.845 & 1 & 0.174 & 3.646 & 0.564 & 23.577 \\
\hline Size & 0.174 & 0.884 & 0.039 & 1 & 0.844 & 1.190 & 0.211 & 6.726 \\
\hline T status & 1.096 & 0.754 & 2.110 & 1 & 0.146 & 2.991 & 0.682 & 13.114 \\
\hline $\mathrm{N}$ status & 1.661 & 0.670 & 6.147 & 1 & $0.013^{a}$ & 5.263 & 1.416 & 19.559 \\
\hline Grade & 0.646 & 0.729 & 0.787 & 1 & 0.375 & 1.909 & 0.458 & 7.964 \\
\hline Weightloss & 0.739 & 0.778 & 0.902 & 1 & 0.342 & 2.094 & 0.456 & 9.616 \\
\hline
\end{tabular}




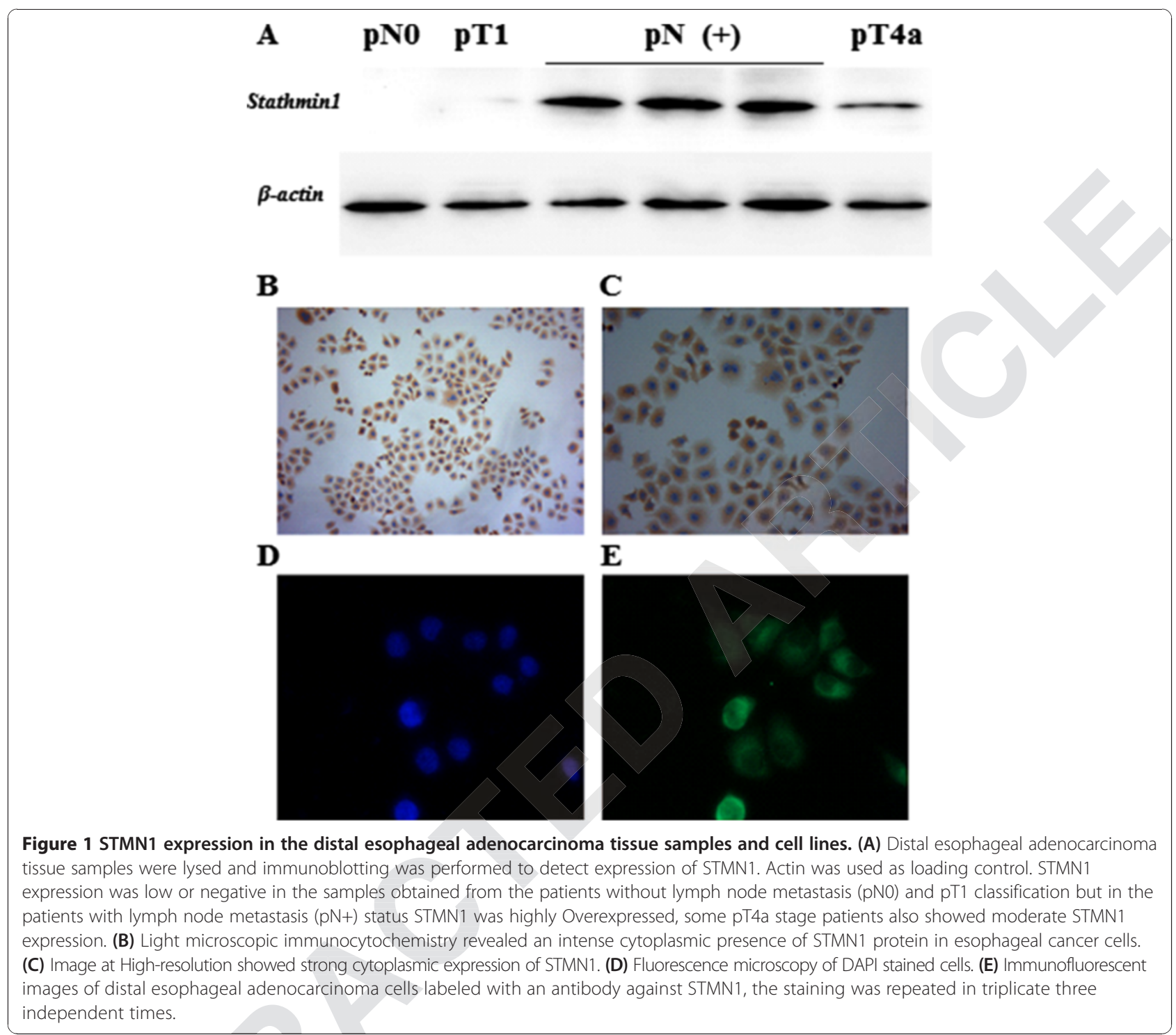

shown in (Figure 2C, D, E, and F) confirmed $>90 \%$ transduction efficiency. To evaluate the silencing efficiency qRT-PCR and western blotting analysis were performed.

\section{Lentivirus-mediated RNAi efficiently suppressed STMN-1} expression in esophageal adenocarcinoma cells

To investigate the role of STMN-1 in esophageal adenocarcinoma cell's growth and metastasis, we constructed lentivirus vector with STMN-1 shRNA and infected esophageal adenocarcinoma cells. Stable expression of STMN-1 shRNAs specifically knocks down STMN-1 expression and activity in esophageal adenocarcinoma cell. After viral infection, more than $80 \%$ of the cells were GFPpositive, indicating a high efficiency of shRNA delivery (Figure 2C, D). The results of the western blot showed that the average band intensities of STMN-1 normalized to actin (Figure 3A).

\section{Stable knockdown of oncogenic STMN-1 by lenti-shRNA significantly inhibited esophageal adenocarcinoma cell's growth in vitro}

To investigate the possible anti-proliferative effects of STMN-1 knockdown in vitro, a CCK-8 assay was performed and a cell growth curve was generated. STMN-1 shRNA transduced esophageal adenocarcinoma cells showed significantly reduced viability relative to Nonsilencing control-transduced cells. STMN-1 knockdown inhibited the proliferation of esophageal adenocarcinoma cells in vitro, indicating that the differential STMN-1 gene expression affects the growth of esophageal adenocarcinoma cells (Figure 4A). 

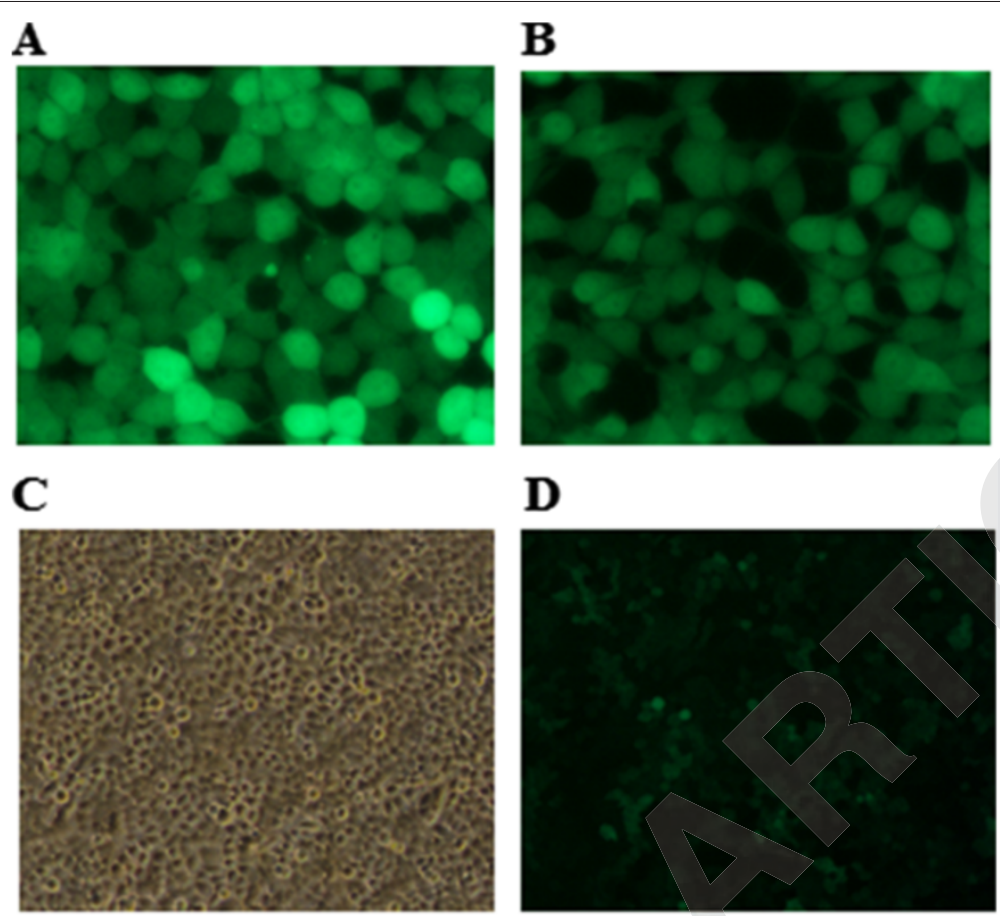

\section{D}
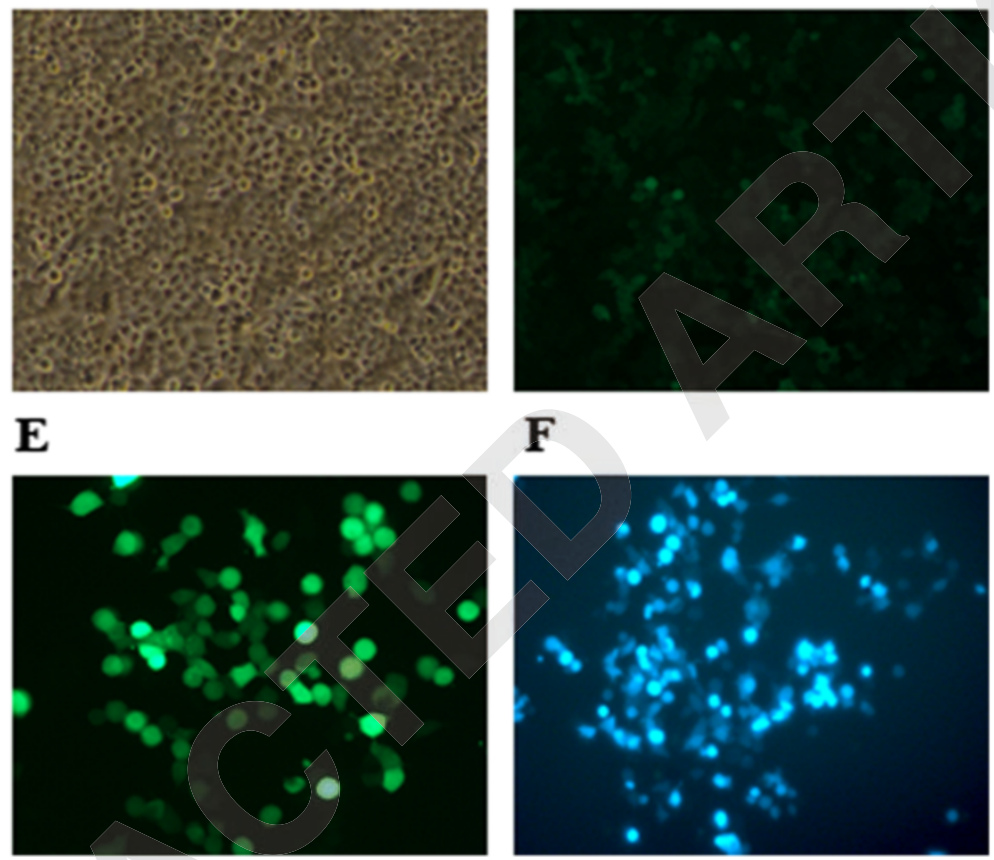

$\mathbf{F}$

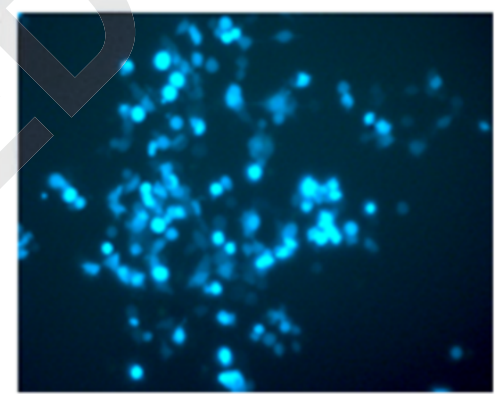

Figure 2 Transfection, Lenti-virus production and transduction. Co-transfection of the Trans-Lentiviral packaging mix with a shRNA transfer vector into HEK293T packaging cells was done using Arrest-In Transfection Reagent. Following co-transfection, replication-incompetent virions were released into the media which were collected after $48 \mathrm{~h}$ and 72 respectively. (A) Fluorescence microscopy image showing TurboGFP expression from the pGIPZ STMN1 vector cells $72 \mathrm{~h}$ post-transfection. (B) Image showing the TurboGFP expression from the GIPZ Non-silencing Control. (C) Phase contrast microscopy image. (D) TurboGFP images showing shRNA delivery efficiency. (E) \& (F) post puromycine selection images showing efficient transduction of Lenti-STMN1 shRNA in distal esophageal cancer cells.

Stable knockdown of STMN-1 expression inhibits migration, invasion and metastasis of esophageal adenocarcinoma cells

Cell migration was evaluated in the Boyden migration assay two days after esophageal adenocarcinoma cells were transfected with Non-silencing shRNA infected cells or STMN1shRNA. STMN-1 shRNA transfected inhibited migration (Figure 5A, B, C, D). To evaluate the function of STMN-1 on Esophageal adenocarcinoma cell invasion, Matrigel invasion chambers were utilized. Inhibited STMN-1 expression led to a significantly decreased invasive ability of esophageal adenocarcinoma cells (Figure 5E).

\section{Knockdown of STMN-1 expression induced cell cycle arrest at the $\mathrm{G} 1 / \mathrm{S}$ phase}

The Flow cytometery results demonstrated that, STMN-1 shRNA treated cells induce an accumulation of esophageal adenocarcinoma cells in the G1/S phase of the cell cycle (Figure 4C, D). In the control group $48.6 \%$ of cells were in the G1 phase and $46 \%$ of the cells were in S of the cell cycle, and 24 post transfection, cells accumulated in G1 $54.4 \%$ but reduced to $40.5 \%$ in S phase. These results demonstrate that knockdown of STMN-1 induces G1/S phase arrest in esophageal adenocarcinoma cells. 


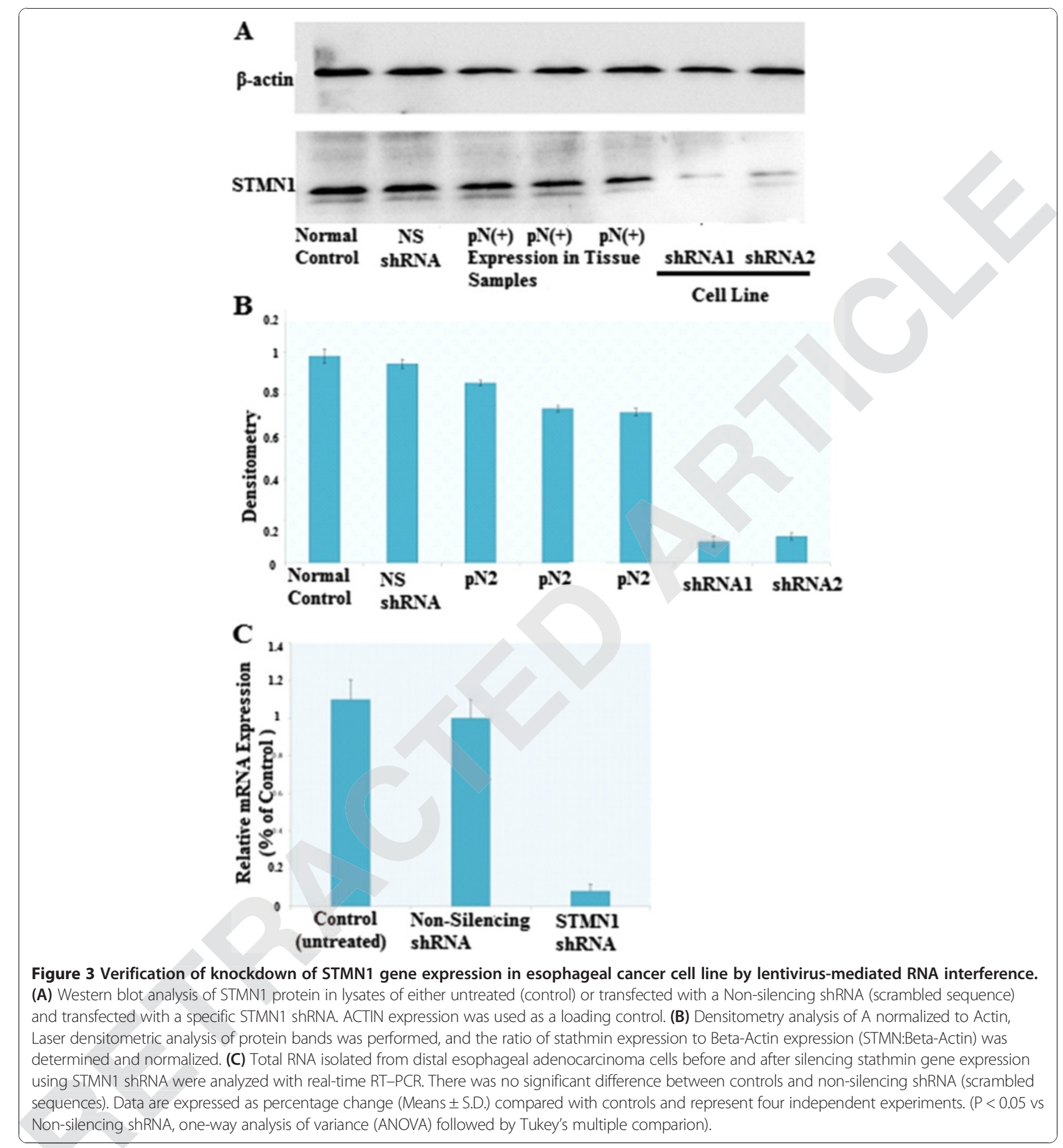

Knockdown of STMN-1 expression induced esophageal adenocarcinoma cells apoptosis

As shown in Figure 4B, the percentage of apoptotic cells infected with STMN-1 shRNA was much higher than that in control shRNA group $(P<0.01)$. No significant difference was found between control shRNA infected cells and non-infected cells. These data indicated that knockdown of STMN-1 expression induced apoptosis in the esophageal adenocarcinoma cells.
In vivo studies of esophageal adenocarcinoma xenograft tumor models in nude mice

To further evaluate the effects of reduced STMN-1 expression on the tumorigenic phenotype and in particular its contribution to in vivo tumor growth. Esophageal adenocarcinoma cells infected with non-silencing shRNA and STMN1 shRNA or untreated were injected into mice, (Figure 6C, D). STMN1 shRNA transfected grew rapidly as compared to negative control. These results 

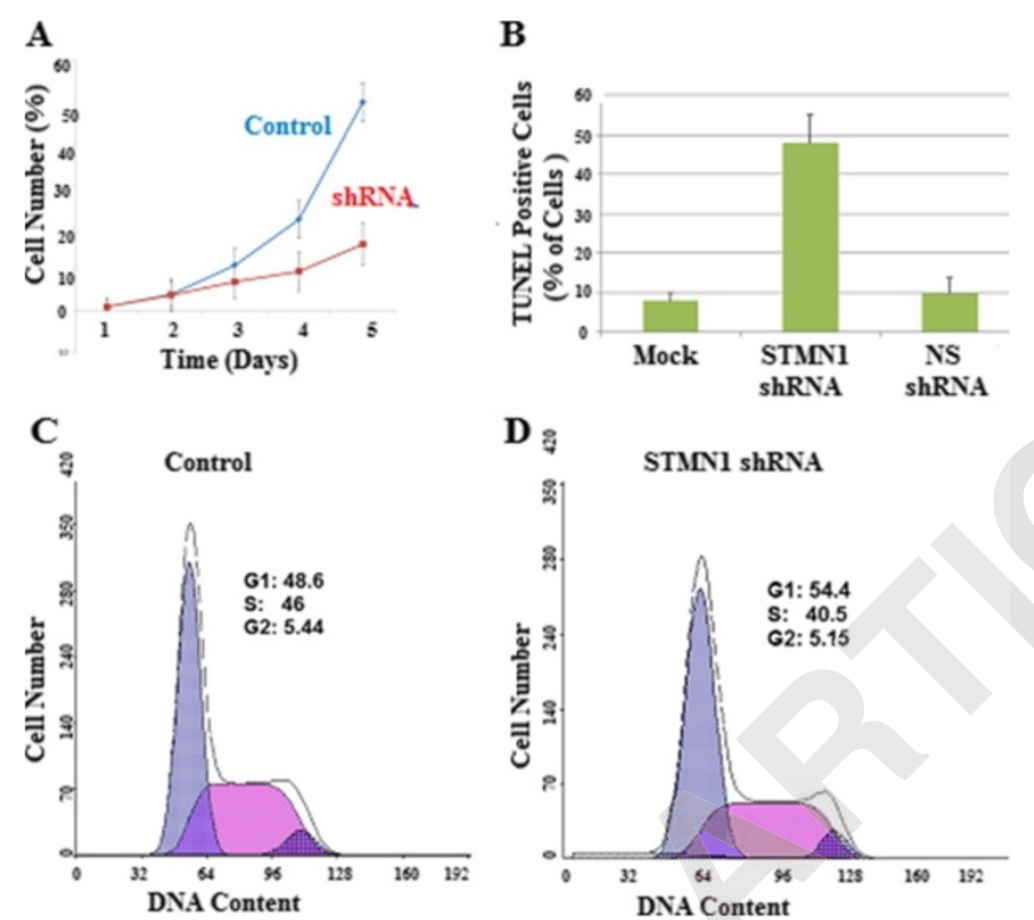

Figure 4 Effects of STMN1 knockdown on cell growth and apoptosis. (A) Cells were transfected with Non-silencing shRNA as a negative control or STMN1shRNA. Cell proliferation was measured by CCK-8. Data are expressed as percentage change (Means \pm S.D.) compared with controls and represent six independent experiments. ( $P<0.05$ vs Non-silencing shRNA, one-way analysis of variance (ANOVA) followed by Tukey's multiple comparion). (B) Apoptosis was assessed by TUNEL assay, the amount of DNA fragmentation (apoptosis) was assessed by TUNEL assay for esophageal cancer cells before and after silencing stathmin gene expression utilizing STMN1 shRNA. There was no significant difference between controls and non-silencing shRNA (scrambled sequences). Experiments were performed in triplicate. (C) Cell-cycle distributions of control shRNA infected cells and (D) STMN1 shRNA infected cells as measured by flow cytometry.

demonstrate that in vivo tumor growth was inhibited by shRNA-mediated knockdown of STMN-1 expression in esophageal adenocarcinoma cells.

\section{Discussion}

Stathmin 1 (STMN-1), also known as p17, p18, p19, $19 \mathrm{~K}$, metablastin, oncoprotein 18, LAP 18 and Op18, is a $19 \mathrm{kDa}$ cytosolic protein. Its function as an important regulatory protein of microtubule dynamics has been well characterized [6]. It has been reported that STMN-1 is overexpressed in many Human malignancies, such as Leukemia, Lymphoma, Neuroblastoma, Ovarian, Prostatic, Breast and Lung cancers [7] and the modulation of its expression correlates with invasion and metastasis. In our study, we found that down regulating STMN-1 by specific shRNA leads to reduction in STMN-1 protein expression in esophageal cancer cells. STMN-1 has been implicated in G1-S checkpoint control of cell cycle progression by influencing the dynamics of microtubule formation and progression of the cell cycle [10]. In human cancers, STMN-1 overexpression is associated with increased malignancy, metastasis formation and decreased patient overall survival suggesting that STMN-1 could serve as a molecular marker to identify patients with more aggressive disease [11]. Recent studies have shown that inhibition of STMN-1 expression in malignant cells interferes with their orderly progression through the cell cycle and abrogates their transformed phenotype [12]. Thus; STMN-1 provides an attractive molecular target for disrupting the mitotic apparatus and arresting the growth of malignant cells.

The protein expression of STMN-1 has been explored and found to correlate to clinicopathologic factors and poor prognosis in several cancers in different tissues such as Brain [13], Oral mucosa [14], Breast [15-17], Urothelial [18] as well as Ovarian [19], and Uterine cervix. So far, the expression of STMN-1 has not been explored in distal esophageal adenocarcinoma. To reveal a possible correlation between STMN-1 up-regulation and lymph node metastasis in distal esophageal adenocarcinoma, we examined the STMN-1 expression. We found that the expression of STMN-1 was positively correlated with lymph node metastasis stages in distal esophageal adenocarcinoma (Tables 1, 2).

In this study, we first investigated the STMN-1 protein expression in esophageal adenocarcinomas tissue $(n=63)$ by western blotting analysis, and showed that STMN-1 was highly expressed in $31(49.21 \%)$ of the 63 distal 

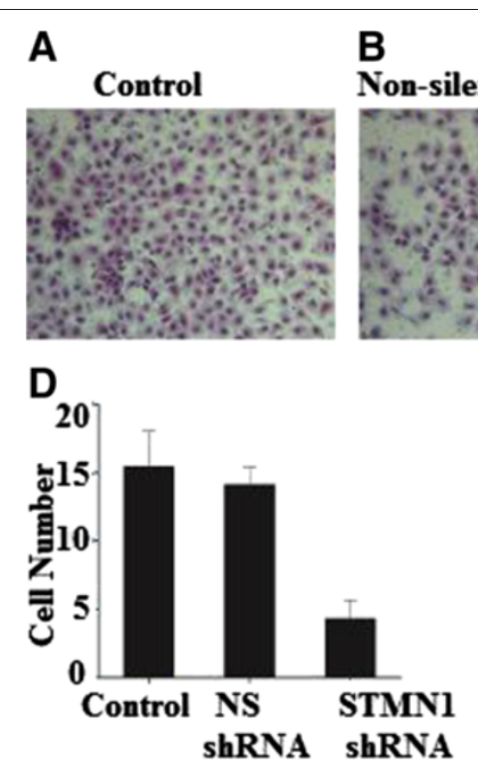

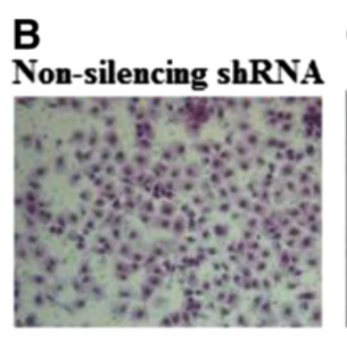

C

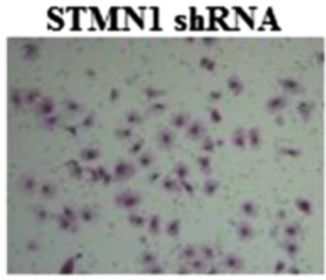

E

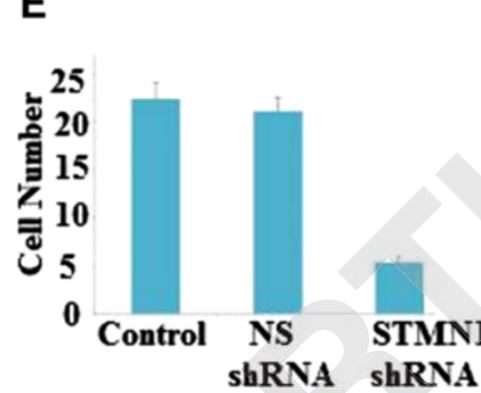

Figure $\mathbf{5}$ Effects of STMN1 knockdown on cell migration and invasion. The images of cells migrating PVPF filters as examined by cell migration assay using Boyden chambers. Cell migration was evaluated in the Boyden migration assay two days after Esophageal cancer cells were either (A) untreated or (B) transfected with Non-silencing shRNA or (C) stathmin1 siRNA. (D) Representing average of STMN1 shRNA infected cells, Non-silencing shRNA infected cells and non-infected cells $(P<0.05)$ versus control. (E) The average invading cell counts of STMN1 shRNA infected cells, Non-silencing shRNA infected cells and non-infected cells. Data are expressed as percentage change (Means \pm S.D.) compared with controls and represent four independent experiments. ( $P<0.05$ vs Non-silencing shRNA, one-way analysis of variance (ANOVA) followed by Tukey's multiple comparion).

esophageal adenocarcinoma cases (Table 1). The level of STMN-1 was significantly higher in esophageal cancer tissue with lymph node metastasis $p \mathrm{~N}(+)$ than $p \mathrm{~N}(-)$. Multivariate logistic regression analysis demonstrates that the STMN-1 overexpression is an independent factor for lymph node metastasis in distal esophageal adenocarcinoma (Table 2). On the basis of clinical research, we found that STMN-1 was highly overexpressed in distal esophageal adenocarcinoma cases with lymph node metastasis and advanced $\mathrm{T}$ classification. This means that this gene can imply an aggressive malignancy of Adenocarcinoma. STMN-1 is up-regulated in many neoplasms and the modulation of its expression correlates with invasion and metastasis [20]. Lymph node metastasis in distal esophageal adenocarcinoma has a worse prognosis. Therefore, it's very important to uncover the necessary biological marker that can predict early lymph node metastasis.

Laboratory research was conducted to correlate our clinical findings. We used shRNA methodology to stably silence the expression of STMN-1 in esophageal adenocarcinoma cells. Independent from its role in cell division, STMN-1 has also been shown to regulate cell motility, enhance cell migration, and promote metastasis [20,21]. We found that stable knockdown of STMN-1 led to reduced proliferation rates and migration in vitro, and decreased in vivo tumorigenicity.
Apoptosis, is a carefully regulated process that can be induced by a variety of stimuli, including programmed tissue remodeling, cell detachment (anoikis), genomic damage, hypoxia, signaling pathway derangement, growth factor or cytokine limitations, and infection [22-24]. Dysregulation of apoptosis contributes to the development of cancer, as decreased rates of cell death facilitate an overall increase in cell number. For this reason, the apoptotic pathway, similar to the cell cycle proteins, has been an attractive target for therapeutic development. Apoptosis is deregulated in many cancers, making it difficult to kill tumors. Drugs that restore the normal apoptotic pathways have the potential for effectively treating cancers that depend on aberrations of the apoptotic pathway to stay alive [25]. Apoptosis was significantly increased in STMN1 shRNA knockdown cells.

RNA interference (RNAi) is a widespread and powerful tool used for the functional regulation of gene expression, and it has been developed as a potential therapeutic strategy in various diseases, including cancers. However, the knockdown effect of regular synthesized siRNA only lasts for a short time and does not allow the stable inhibition of target gene function. At the present time, for efficient delivery and stable knockdown of target genes, lentiviral vectors are the most appealing tool. The lentiviral vectors for shRNA expression were used in our system. This 

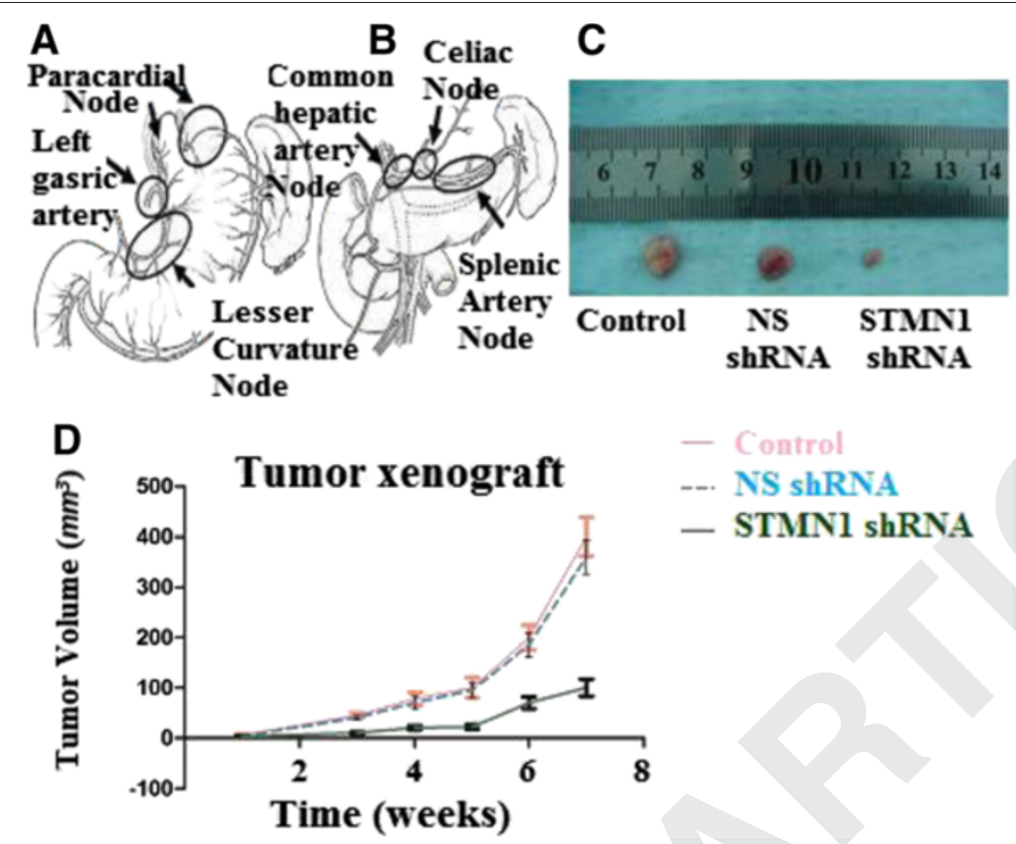

Figure 6 Abdominal lymph node clearance and xenograft tumor models in nude mice. (A) Perigastric lymph nodes, lymph nodes along the left gastric artery and lesser curvature lymph nodes dissection. (B) Distant abdominal lymph node dissection. Abdominal Esophagus drains into superior gastric artery, celiac axis, common hepatic artery and splenic artery lymph nodes. (C) Esophageal cancer cells were either untreated or transfected with Non-silencing shRNA (scrambled sequence) as a negative control and transfected with STMN1 shRNA were xenografted subcutaneously in the BALB/c-nu/nu male mice. Tumor mass (xenograft) volume was measured every week from week 3 to week 7. Data are expressed as percentage change (Means \pm S.D.) compared with controls and represent four independent experiments. ( $P<0.05$ vs Non-silencing shRNA, one-way analysis of variance (ANOVA) followed by Tukey's multiple comparion). (D) Photograph of xenografts dissected from nude mice after 7 weeks subcutaneous inoculation showing suppression growth of cancer cells transfected with stathmin 1 shRNA as compared to cells transfected with untreated or transfected with Non-silencing shRNA.

approach allows for the stable suppression of target gene expression both in cell culture conditions and in animals.

STMN-1 increases the proliferation rate of leukemia cells [26]. Our results showed that STMN-1 was directly involved in cell proliferation and adhesion of esophageal adenocarcinoma cells. STMN-1 expression in oral cancer found to be correlated with tumour progression and poor prognosis [14]. STMN-1 Up regulation increases the aggressiveness of esophageal adenocarcinoma cells, and enhances the migration ability which is responsible for metastasis.

To validate effectiveness of down regulation of STMN-1 expression, we performed in vivo studies in nude mice; our results showed that STMN-1 shRNA could effectively down regulated STMN-1 expression by up to $85 \%$ in esophageal adenocarcinoma cells with great specificity (Figure 6C) indicating that the lentiviral-based RNAi, because of its potency, could be utilized as an effective strategy for cancer therapy. Additionally, the blockage of proliferation in esophageal cell lines and the inhibition of tumorigenesis in nude mice support the effectiveness of this strategy.
STMN-1 is an indicator of lymph node metastasis in distal esophageal adenocarcinomas and its silencing reverses the malignant biological behavior in esophageal adenocarcinoma cells. These results suggest that expression of STMN-1 oncogene product may be associated with the biological aggressiveness of adenocarcinomas of the distal esophagus.

The results we describe herein provide new insights for applying STMN-1 as a potential target for antitumor therapy by silencing its expression with STMN-1 shRNA. Cancer targets can be exploited by different strategies. Thus far, the more successful clinical approaches have been achieved with small-molecule drugs, monoclonal antibodies, and protein kinase inhibitors. Of the new anticancer drugs approved by the U.S. Food and Drug Administration (FDA) since 2000, fifteen have been targeted drug therapies, compared with only five traditional chemotherapeutic agents. One of the promising emerging technologies is RNAi, which is now being vigorously developed [27]. Current data from initial clinical trials indicate that RNAi drugs will soon provide another potent class of agents against malignant diseases [28]. The pharmacologic validation of STMN-1 as a 
therapeutic target has not been fully elucidated because of the lack of research in this area. Based on in vitro and in vivo studies the shRNA targeting of STMN-1 has shown to inhibit cell proliferation in vitro and tumor growth in vivo. The potential outcome of STMN-1 inhibition in cancer therapy is promising,

\section{Conclusion}

Our clinical data shows that increased STMN-1 expression is associated with more aggressive disease, Thus, STMN-1 is an indicator of lymph node metastasis in distal esophageal adenocarcinomas and it has oncogenic effects in cell lines and xenografts. The differential expression status of STMN-1 between the early and advanced $\mathrm{pN}$ stages of distal esophageal adenocarcinoma may provide insights into the process of tumorigenicity and for planning new treatment strategies.

In summary, the results of the current study show that using RNAi to reduce STMN-1 levels in esophageal cancer cells significantly reduced the expression of STMN-1 protein, cellular proliferation, migration, invasion, in vitro and tumor growth in vivo. Further investigations are required to test which routes of delivery (e.g., direct intratumoral injections or systemic administration) of STMN-1 shRNA would be most efficient for esophageal cancer therapy.

\section{Methods}

\section{Ethics statement}

The study protocol was approved by the Ethics Committee of Provincial Hospital Affiliated to Shandong University. All the participants provided their written informed consent for inclusion in the data analysis and manuscript publication. Data were analyzed anonymously.

\section{Patient selection and distal esophageal adenocarcinoma specimen}

From June 2010 to June 2012, sixty-three eligible patients with previously untreated, potentially resectable, clinical stage I-III distal esophageal adenocarcinomas were admitted to the Department of Thoracic Surgery, Provincial Hospital Affiliated to Shandong University, China. These patients were all diagnosed with adenocarcinoma of the distal esophagus. Complete clinical data, along with their medical histories was kept in separate files for each patient enrolled in the study. None of the patients had received chemotherapy or radiotherapy prior to surgery. Informed consent to obtain tissue samples for the study was taken from each patient. Tissue samples were obtained at the time of surgical resection. There were forty-five men and eighteen women, and the median age was fifty-seven. The age range was thirty-four to seventy-nine years old. To define the stage of adenocarcinoma of the distal esophagus, we used TNM classification according to the 2009 UICC standard [4].

\section{Mice}

BALB/C-nu/nu male mice (weight 18-22 g, 6 weeks old) were obtained from the Shanghai Tumor Institution.

All experimental procedures using animals in the present study had received prior approval by the Institutional Animal Care and Use Committee of Shandong University under Contract 2011-0015.

\section{Cell lines, culture medium and reagents}

Human Embryonic Kidney 293 cells (HEK93T) were obtained from the Shanghai Tumor Institution. Human esophageal adenocarcinoma cells were kindly provided by the pathology department of provincial hospital. Classical Liquid Media Dulbecco's Modified Eagles Medium (DMEM), High Glucose RPMI Media 1640 was purchased from HyClone (Thermo Scientific). Fetal bovine serum (FBS) was purchased from Gibco (Invitrogen Co., USA). Dimethyl sulfoxide (DMSO) was purchased from SigmaAldrich (St Louis, Missouri, USA). Rabbit Anti-STMN1 Polyclonal antibody and HRP-conjugated secondary antibodies were purchased from Abcam (Cambridge, MA).

\section{Operation method}

Patients were operated through the 7 th intercostal space with left posterolateral thoracotomy. The lower esophagus was mobilized and periesophageal lymph nodes were cleared. Thoracoabdominal two-field lymph node dissection was undertaken. An incision was made through the diaphragm to enter the abdominal cavity. The greater and lesser curvatures were mobilized. We routinely removed perigastric lymph nodes, including left gastric artery node (Figure 6A). For the nodal stations around the celiac trifurcation, we performed selective dissection when the swollen lymph nodes were found or touched, and sometimes we only performed lymph node sampling when no obviously swollen one was found (Figure 6B). From the upper and lower margin of the tumor more than $5 \mathrm{~cm}$ esophageal and gastric tissue were resected and then Gastro-esophageal anastomosis was made mechanically at the level of the inferior pulmonary vein. The average age in this group was fifty-seven. This operation was performed on forty-five males and eighteen females. The average lymph node clearance was $21.5 \%$ per patient.

\section{Cell culture}

Esophageal adenocarcinoma cells were grown in RPMI 1640 medium supplemented with $10 \%$ fetal bovine serum (FBS) and 100 units/ml penicillin, and $100 \mathrm{mg} / \mathrm{ml}$ streptomycin. The cells were grown at $37^{\circ} \mathrm{C}$ in a humidified atmosphere containing 5\% CO2. Stock cultures of each cell 
line were routinely sub-cultured at least once a week and the medium was changed every $2-3$ days.

\section{Lentivirus-mediated short hairpin RNA (shRNA) knockdown of gene expression}

To knockdown STMN-1 expression, pGIPZ-lentiviral shRNAmir vectors targeting human STMN1 and Nonsilencing pGIPZ control vector were purchased from Open Biosystems (Thermo Fisher Scientific, Inc.). The sequences of STMN-1 shRNA are as following: $5^{\prime}$-TTAT TAGCTTCCATTTTGT-3'; 5' -TCTCTTCTATTGCCTT CTG-3' and 5' -TTATTAACCATTCAAGTCC-3' . Lentiviral shRNA was produced by Co-transfection of the TransLentiviral packaging mix with a shRNA transfer vector into HEK293T packaging cells (OpenBiosystems). For cell infection, viral supernatants were supplemented with $6 \mu \mathrm{g} / \mathrm{mL}$ polybrene and incubated with cells for 24 hours. Esophageal adenocarcinoma cell lines were transduced by the lentiviral particles followed by puromycin selection $(1 \mu \mathrm{g} / \mathrm{mL})$ for 10 days. The cells stably expressing shRNA were maintained in puromycin $(0.2 \mu \mathrm{g} / \mathrm{mL})$.

\section{RNA extraction and qRT-PCR}

Total RNA extraction was performed using Trizol reagent (Invitrogen) according to the manufacturer's instruction. RNA concentration was measured by Nano Drop 1000 (Thermo Fisher Scientific). One microgram of total RNA extracted from the cells was subjected to reverse Transcription (RT). Verso cDNA Ki (Thermo Scientific) was used for cDNA synthesis. Real-time RT-PCR was used to quantify the expression level of STMN-1 gene in Esophageal adenocarcinoma cell line using ABI 7300 real-time PCR thermal cycle instrument (ABI, USA), according to the supplied protocol. Amplification conditions were as follows: Reverse-transcription reaction: $42^{\circ} \mathrm{C}, 30$ minutes per cycle. PCR cycling conditions were as follows: Enzyme activation $95^{\circ} \mathrm{C} 15$ minutes per cycle, denaturation $95^{\circ} \mathrm{C}$ at 15 seconds per 40 cycles and Annealing/Extension at $60^{\circ} \mathrm{C}$ for 60 seconds.

A Real-time PCR reaction was performed using the Solaris qPCR Gene Expression Master Mix with LOW ROX premixed and $1 \mu \mathrm{L}$ of total cDNA in each well, Stathmin specific primers were as follows:

(F, AGAATACACTGCCTGTCGCTTG; R, AGGCAC GCTTCTCCAGTT). The relative expression levels were normalized to expression of endogenous Beta-Actin. (Primers: F, TGGAGAAAATCTGGCACCAC; R, GGTCTCAAA CATGATCTGG).

\section{Protein extraction and western blotting}

For whole-cell protein extraction, esophageal adenocarcinoma cell line were washed with cold PBS and subsequently lysed in cold RIPA lysis buffer (50 mM Tris-HCl, pH 7.4, $150 \mathrm{mM} \mathrm{NaCl}, 1 \mathrm{mM}$ dithiothreitol [DTT], 0.25\% sodium deoxycholate, $0.1 \%$ NP-40) containing $1 \mathrm{mM}$ phenylmethysulfonyl fluoride (PMSF), $50 \mathrm{mM}$ sodiumpyrophosphate, $1 \mathrm{mM}$ Na3VO4, 1 mM NaF, 5 mM EDTA, 5 mM EGTA, and protease inhibitors cocktail (Roche). Cell lysis was performed on ice for 30 minutes. Clear protein extracts were obtained by centrifugation for 30 minutes at $4^{\circ} \mathrm{C}$. Protein concentrations were determined by the method of Bradford using the Bio-Rad protein assay reagent (Bio-Rad) and 20-40 mg of protein mixed with loading buffer was loaded per lane, separated by $12 \%$ SDSpolyacrylamide gel electrophoresis (SDS-PAGE). Proteins were transferred to PVDF membrane filters (Millipore, USA). Nonspecific binding was blocked by incubation in phosphate-buffered saline (PBS) containing 0.1\% Tween 20 (PBS-T) and 5\% skim milk. PVDF membranes were blocked with $5 \%$ dry milk for one hour at $4^{\circ} \mathrm{C}$ Membranes were incubated in STMN-1 primary antibody (1:1000) overnight at $4^{\circ} \mathrm{C}$. The membranes were then incubated with the corresponding secondary antibody (1:2000, horseradish peroxidase-conjugated anti-rabbit) in TBST-5\% nonfat milk for 1 hour at room temperature and the immunoreactive bands were visualized using EZ ECL Chemiluminescence Detection Kit for HRP (Biological Industries Ltd, Israel). Images were acquired using the LAS3000 Imager (Fujifilm). Membranes were re-probed for Beta-Actin as a loading control.

\section{Cell proliferation assay}

Cell Counting Kit-8 (CCK-8; Dojindo) was used in cell proliferation assay. 3000 viable cells per well into 96-well tissue culture plates in a final volume of $100 \mu \mathrm{l}$. Every 24 hours, a plate was subjected to assay by adding $10 \mu \mathrm{l}$ of CCK-8 solution to each well, and the plate was further incubated for 4 hours at $37^{\circ} \mathrm{C}$. The absorbance at $450 \mathrm{~nm}$ was measured with a micro plate reader. The experiment was performed in eight replicates.

\section{Adhesion assay}

Cell adhesion was analyzed using a Vybrant Cell Adhesion Assay kit (Molecular Probes, USA) was used according to the manufacter's instructions. Briefly, cells in suspension were labeled with Calcein AM dye, and equal numbers of cells were plated in 96-well plates. At timed intervals, non-adherent cells were removed and adherent cells were quantified with the use of a SAFIRE fluorescence plate reader.

\section{Migration and invasion assays}

For trans-well migration assay, 50,000 cells were added to upper chamber in serum free media and migration at $37^{\circ} \mathrm{C}$ towards $10 \%$ FBS containing growth media was determined either after 24 hours or 48 hrs. Cells migrated through the membrane were fixed, stained with $H \& E$ (Sigma) and counted under light microscope. For invasion 
assay, lower chambers of matrigel coated invasion plates were coated with $10 \mathrm{mg} / \mathrm{ml}$ fibronectin overnight at $4^{\circ} \mathrm{C}$ and cells invading through matrigel were fixed and stained after 48 hours.

\section{TUNEL assay}

Terminal deoxynucleotidyl transferase-mediated dUTP nick end labeling (In Situ Cell Death Detection Kit POD) was used for proving apoptosis in individual cells. The TUNEL working procedure was carried out following the producer's directions (Roche). Endogenous peroxidase was blocked by incubation in $1.3 \% \mathrm{H} 2 \mathrm{O} 2$ in PBS for $10 \mathrm{~min}$ at room temperature before enzymatic labeling. During the TUNEL procedure samples were washed in PBS. The fluorescent signal conversion using anti-fluorescence antibody conjugated with peroxidase and substrate color reaction applying chromogen DAB (3,3'-diaminobenzidine tetrahydrochloride; Sigma-Aldrich, Germany) were proceeded after enzymatic labeling.

\section{Flow cytometric cell cycle analysis}

For cell cycle analysis esophageal adenocarcinoma cells were collected in twenty-four post transfection, $6 \mathrm{~cm}$ plates. Cells were harvested using cold PBS and fixed in 70\% cold ethanol overnight in $4^{\circ} \mathrm{C}$ and treated with $1 \mathrm{ng} / \mathrm{ml}$ RNase A for 10 minutes at $37^{\circ} \mathrm{C}$. Cellular DNA was stained with $15 \mathrm{ng} / \mathrm{ml}$ propidium iodide (PI) for 30 minutes at $37^{\circ} \mathrm{C}$ in the dark. The cells then were sorted by FACS Calibur Flow Cytometer (Becton Dickinson, CA) and cell-cycle profiles were determined using the ModFitLT software (Becton Dickinson, SanDiego, CA). The experiments were repeated on two separate occasions.

\section{Immunocytochemistry}

Stably transfected esophageal adenocarcinoma cells were seeded into 4-chambered glass slides (Nunc Lab-Tek Chamber Slide System). Cells were then incubated overnight. After 24 hours, cells were rinsed with PBS, fixed with $3.7 \% \mathrm{w} / \mathrm{v}$ paraformaldehyde (Sigma), rinsed with PBS, and permeablized in $0.5 \%$ Triton X-100 (Sigma). Nonspecific immunoglobulin binding was blocked with 5\% normal goat serum and $0.5 \%$ NP-40 (Sigma). Primary antibodies recognizing with STMN-1 (abicam) were diluted 1:100 in blocking solution. After incubation with primary antibody, cells were rinsed with $0.05 \%$ Tween-20 (Bio-Rad) in PBS, and then incubated with secondary antibody for $1 \mathrm{~h}$ at RT. Stain with 3,3'-Diaminobenzidine (DAB) and observed under light microscope.

\section{Immunofluorescence}

To examine the protein expression of STMN-1 gene, we performed immunofluorescence analysis. Briefly, the cells were washed with PBS and fixed in 4\% paraformaldehyde for 10 minutes at $37^{\circ} \mathrm{C}$ followed by absolute methanol for 10 minutes at $4^{\circ} \mathrm{C}$ and blocked in PBS containing $1 \%$ skimmed milk for 10 minutes. The samples were then incubated with affinity-purified Rabbit Anti-Human STMN-1 Polyclonal Antibody (Abcam, USA) at a dilution of 1: 100 for 2 hours, rinsed twice with PBS, and incubated with goat anti-rabbit IgG Alexa Fluor 488 (Molecular Probes) for 1 hour.

\section{In vivo studies of esophageal adenocarcinoma cell line xenograft tumor models in nude mice}

Six-week-old male BALB/c nude mice were housed in a temperature-controlled, pathogen-free animal facility with 12-hour light and dark cycles. Mice were injected subcutaneously into bilateral flanks with untransfected cells, or transfected with Non-silencing shRNA and STMNEshRNA $\left(2 \times 10^{6}\right.$ cells in $200 \mu \mathrm{l}$ PBS) to establish tumors. Tumor mass (xenograft) volume was measured every week from week 3 to week 7 . After 7 week mice were sacrificed, and tumors were harvested.

\section{Statistical analysis}

For comparison of more than three groups, we used oneway analysis of variance, followed by Tukey's multiple comparison-values $<0.05$ were considered statistically significant. One-way analysis of variance (ANOVA), followed by the LSD post hoc test was used to compare mean differences in 2 or more groups. Associations between categorical variables were evaluated by Pearson's chi-square test. $\mathrm{P}$ values represent 2 - sided tests and are of statistical significance when $P<0.05$. These statistically significant variables were found in the univariate analysis $(P, 0.05)$, and the Logistics Regression Model. Similar ratio statistics were employed to further evaluate them for multivariate. All statistical analysis was performed by using IBM SPSS version 20.0

\section{Competing interests}

The authors hereby declare that they have no competing interests.

\section{Authors' contributions}

JA was involved in the design of the study, acquisition of data, helped in the collection of clinical data, performed the molecular tests and statistical analysis and drafted the manuscript. JA and YC performed laboratory experiments, analyzed and interpreted the data. LHJ and LCS enrolled the study patients in the department, collected the biological samples, collected the clinical data and made a critical review of the manuscript. SYL conceived the study and was involved in its design, supervised the study. WZ helped to analyze the data, participated in the statistical analysis and revising it critically for important intellectual content. All authors read and approved the final manuscript.

\section{Acknowledgments}

We are grateful to Renee Lovett for English language editing of our manuscript. This study was supported by a grant from the Natural Science Foundation of Shandong Province of China (Y2007C102), the Medical Science and Technology Development Foundation of Shandong Province of China (2007H2071), Key research Project from Shandong Science Technology Commission (25006GG2202025) 


\section{Author details}

${ }^{1}$ Department of Thoracic Surgery, Provincial Hospital Affiliated to Shandong University, 250021 Shandong, China. ²Division of Gastrointestinal Surgery, Provincial Hospital Affiliated to Shandong University, 250021 Shandong, China. ${ }^{3}$ Department of Nephrology, Provincial Hospital Affiliated to Shandong University, Shandong 250021 P R China.

Received: 11 August 2013 Accepted: 15 January 2014

Published: 16 January 2014

\section{References}

1. Jemal A, Siegel R, Xu J, Ward E: Cancer statistics. CA Cancer J Clin 2010, 60:277-300.

2. Jemal A, Bray F, Center MM, Ferlay J, Ward E, Forman D: Global cancer statistics. CA Cancer J Clin 2011, 61:69-90.

3. Kachroo S, Etzel CJ: Decreasing the cancer burden in developing countries: concerns and recommendations. Eur J Cancer Care (Engl) 2009, 18:18-21.

4. Sobin LH, Gospodarowicz MK, Wittekind C: TNM Classification of Malignant Tumours. 7th edition. Oxford, UK: Wiley-Blackwell; 2009.

5. AJCC Cancer Staging Handbook: ftACSM. 7th edition. New York, NY: Springer; 2010:129-143.

6. Sobel A: Stathmin: a relay phosphoprotein for multiple signal transduction. Trends Biochem Sci 1991, 16:301-305

7. Rana S, Maples PB, Senzer N, Nemunaitis J: Stathmin 1: a novel therapeutic target for anticancer activity. Expert Rev Anticancer Ther 2008, 8:1461-1470.

8. Mistry SJ, Li H-C, Atweh GF: Role for protein phosphatases in the cellcycle-regulated phosphorylation of stathmin. Biochem J 1998, 334:23-29.

9. Singer $S$, Ehemann V, Brauckhoff $A$, Keith $M$, Vreden $S$, Schirmacher $P$, Breuhahn K: Protumorigenic overexpression of stathmin/Op18 by gain-of-function mutation in p53 in human hepatocarcinogenesis. Hepatology 2007, 46:759-768

10. Sherbet GV, Cajone F: Stathmin in cell proliferation and cance progression. Cancer Genomics Proteomics 2005, 2:227-238.

11. Belletti B, Baldassarre G: Stathmin: a protein with many tasks. New biomarker and potential target in cancer. Expert Opin Ther Targets 2011 15:1249-1266.

12. Mistry S, Atweh G: Role of stathmin in the regulation of the mitotic spindle: potential applications in cancer therapy. Mt Sinai J Med 2002, 69:299-304.

13. Kuo M-F, Wang H-S, Kuo Q-T, Shun C-T, Hsu H-C, Yang S-H, Yuan R-H: High expression of stathmin protein predicts a fulminant course in medulloblastoma. J Neurosurg Pediatr 2009, 4:74-80.

14. Kouzu Y, Uzawa K, Koike H, Saito K, Nakashima D, Higo M, Endo Y, Kasamatsu A, Shiiba M, Bukawa $H$, et al: Overexpression of stathmin in oral squamous-cell carcinoma: correlation with tumour progression and poor prognosis. Br J Cancer 2006, 94(5):717-723.

15. Saal LH, Johansson P, Holm K, Gruvberger-Saal SK, She QB, Maurer M, Koujak S, Ferrando AA, Malmström P, Memeo L, et al: Poor prognosis in carcinoma is associated with a gene expression signature of aberrant PTEN tumor suppressor pathway activity. Proc Natl Acad Sci U S A 2007, 2;104:7564-7569.

16. Golouh R, Cufer T, Sadikov A, Nussdorfer P, Usher PA, Brünner N, Schmitt M, Lesche R, Maier S, Timmermans M, et al: The prognostic value of Stathmin-1, S100A2, and SYK proteins in ER-positive primary breast cancer patients treated with adjuvant tamoxifen monotherapy: an immunohistochemical study. Breast Cancer Res Treat 2008, 110:317-326.

17. Brattsand G: Correlation of oncoprotein 18/stathmin expression in human breast cancer with established prognostic factors. Br J Cancer 2000 83:311-318.

18. Lin WC, Chen SC, Hu FC, Chueh SC, Pu YS, Yu HJ, Huang KH: Expression of stathmin in localized upper urinary tract urothelial carcinoma: correlations with prognosis. Urology 2009, 74:1264-1269.

19. Su D, Smith SM, Preti M, Schwartz P, Rutherford TJ, Menato G, Danese S, Ma S, Yu H, Katsaros D: Stathmin and tubulin expression and survival of ovarian cancer patients receiving platinum treatment with and without paclitaxel. Cancer 2009, 1;115:2453-2463.
20. Baldassarre $G$, Belletti B, Nicoloso MS, Schiappacassi M, Vecchione A, Spessotto P, Morrione A, Canzonieri V, Colombatti A: p27(Kip1)-stathmin interaction influences sarcoma cell migration and invasion. Cancer Cell 2005, 7:51-63.

21. lancu-Rubin C, Atweh GF: p27(Kip1) and stathmin share the stage for the first time. Trends Cell Biol 2005, 15:346-348.

22. Adams J, Cory S: The bcl-2 apoptotic switch in cancer development and therapy. Oncogene 2007, 26:1324-1337.

23. Adams JM: Ways of dying: multiple pathways to apoptosis. Genes Dev 2003, 17:2481-2495.

24. Danial NN, Korsmeyer SJ: Cell death: critical control points. Cell 2004 116:205-219.

25. Fesik SW: Promoting apoptosis as a strategy for cancer drug discovery. Rev Cancer 2005, 5:876-885

26. Jeha S, Luo X-N, Beran M, Kantarjian H, Atweh GF: Antisense RNA inhibition of phosphoprotein p18 expression abrogates the transformed phenotype of leukemic cells. Cancer Res 1996, 56:1445-1450.

27. Gewirtz AM: On future's doorstep: RNA interference and the pharmacopeia of tomorrow. J Clin Invest 2007, 117:3612-3614

28. Grimm D: Small silencing RNAs: state-of-the-art. Adv Drug Deliv Rev 2009, 61:672-703

doi:10.1186/1471-2407-14-28

Cite this article as: Akhtar et al.: STMN-1 is a potential marker of lymph node metastasis in distal esophageal adenocarcinomas and silencing its expression can reverse malignant phenotype of tumor cells. BMC Cance 2014 14:28

\section{Submit your next manuscript to BioMed Central and take full advantage of:}

- Convenient online submission

- Thorough peer review

- No space constraints or color figure charges

- Immediate publication on acceptance

- Inclusion in PubMed, CAS, Scopus and Google Scholar

- Research which is freely available for redistribution 